\title{
A Prospective Study on the Clinical Features of Hyponatremic Dehydration in Acute Gastroenteritis
}

\author{
Mohammed R. ${ }^{1}$, Khan M.A. ${ }^{2}$ \\ ${ }^{1}$ Dr. Rashwan Mohammed, Assistant Professor, ${ }^{2}$ Dr M. Ahmedullah Khan, Assistant Professor, both authors are affiliated \\ with Department of Pediatrics, Princess EsraHospital,Deccan College of Medical Sciences, Hyderabad, Telangana. India. \\ Corresponding Author: Dr. M. Ahmedullah Khan, Assistant Professor, Department of Pediatrics, Princess Esra \\ Hospital, Deccan College of Medical Sciences, Hyderabad, Telangana. India. E- mail: ahmedkhan67353@gmail.com
}

\begin{abstract}
Introduction: Babies and children with gentle lack of hydration regularly have negligible or no clinical changes other than a lessening in pee yield. Alongside diminished pee yield in kids with moderate drying out regularly have dried mucous layers, diminished skin turgor, crabbiness, tachycardia with diminished hair like refill, and profound breath. Methodology: The study was done in Deccan College of Medical Sciences \& Owaisi Group of Hospitals. Study population consisted from Infants to children aged till 12 years attending the paediatric Department both OPD and IPD care for acute gastroenteritis are enroll in the study. Aims and Objectives: To estimate the incidence of hyponatraemic dehydrationin neonates and children. To evaluates the clinical features associated with acute gastroenteritis associated dehydration. Results: 80 cases were studied and analyzed to detect Gastroenteritis associated dehydration and its clinical features as a possible risk factor for hyponatremia. It was found that $60 \%$ of the males were having acute gastroenteritis while it was $40 \%$ in female subjects. The data also reveals that higher incidence of acute GE was noted between 6 to 24 months of age while the lower incidence was found in the subjects above 36 months of age. Conclusion: The clinical impression of the type of dehydration and electrolyte disturbances was fairly consistent with serum electrolytes values. This suggest that routine estimation of serum electrolytes is not necessary however it is necessary whenever electrolyte imbalance is suspected on clinical grounds and in cases which do not respond satisfactorily with routine fluid electrolyte therapy.
\end{abstract}

Keywords: Hyponatremia, Gastroenteritis, Dehydration, Vomiting, Diarrhea.

\section{Introduction}

The investigations on recurrence and epidemiological attributes of side effect have appeared at be the main commonest arrangement variation from the norm in hospitalized wiped out grown-ups. In them, it's constantly identified with hypo-osmolality and conventional affiliation and is ascribed to disorder of wrong enemy of diuretic inner discharge emission (SIADH) or wiped out cell disorder. The information on the recurrence of side effect in debilitated youths is kept exclusively to some particular Infection, directing routine support liquids, that are commonly hypo-tonic saline and D5, could intensify the condition. Since a diminishing in sodium grouping of plasma is by all accounts a run of the mill occurrence in hospitalized patients, it's basic that analyzed kids are in risk of side effect should know at the most punctual [1].

Manuscript received: $2^{\text {nd }}$ April 2019

Reviewed: $10^{\text {th }}$ April 2019

Author Corrected: $16^{\text {th }}$ April 2019

Accepted for Publication: $20^{\text {th }}$ April 2019
The occurrence of hyponatremia has been exhibited to be an autonomous hazard factor for expanded mortality in medical clinic inpatients. As hyponatremia is the most well-known electrolyte unsettling influence experienced in clinical drug, it is fundamental that specialists and medical attendants realize how to properly deal with this condition.Extreme hyponatremia has for quite some time been perceived to be related with antagonistic results [2].

Babies and children with gentle lack of hydration regularly have negligible or no clinical changes other than a lessening in pee yield. Alongside diminished pee yield in kids with moderate drying out regularly have dried mucous layers, diminished skin turgor, crabbiness, tachycardia with diminished hairlike refill, and profound breath. A methodical audit of the exactness of clinically foreseeing at any rate 5\% lack of hydration in kids found abnormal skin surface, and decreased 


\section{Original Research Article}

respiratory example to be the best indicators [3]. No ongoing investigations exist with respect to the electrolyte unsettling influences happening in a youngster experiencing AGE. Like wise, there are no particular examinations portraying the clinical highlights related with Hyponatremic drying out, and the pieces of information to separating it from Isonatremic drying out [4].

Despite the fact that drugs are a typical reason for hyponatraemia, different causes ought to likewise be considered. Surveying the patient's liquid status and plasma osmolality can help in finding the reason. As hyponatraemia is regularly connected with liquid maintenance the osmolality is generally diminished, anyway different causes might be related with typical or expanded osmolality [5]. In neonate, hypernatremic dehydration might be associated as a weight reduction with over $10 \%$ of birth weight toward the finish of first seven day stretch of life or if there is clinical discoveries of lack of hydration with hypernatremia. Hypernatremic dehydration is a possibly deadly condition in neonate which unfavorably influences focal sensory system, prompting destroying outcomes like intracranial discharge, thrombosis, and even demise [6].

In spite of these examinations utilizing distinctive techniques for evaluating perception, all reliably discovered that intellectual impedance happens in patients with interminable hyponatremia. Be that as it may, the components clarifying this affiliation stay indistinct. In constant hyponatremia, serum sodium levels decrease bit by bit, permitting the body time to adjust. To anticipate swelling at first, the glial cells utilize the $\mathrm{Na}+\mathrm{K}+-\mathrm{ATPase}$ framework to move sodium out of cells while likewise removing osmolytes [7]. The predominance of serious hyponatremia (serum sodium level under $125 \mathrm{mEq}$ for every $\mathrm{L}$ ) was $4.5 \%, 0.8 \%$, and $10.3 \%$, separately. It is assessed that hyponatremia happens in $4 \%$ to $7 \%$ of the wandering populace, with rates of $18.8 \%$ in nursing homes [8].

Both hyponatremic and hypernatremic patients are usually experienced in a wide assortment of clinical circumstances. Most noticeable among the clinical signs of both of these electrolyte variations from the norm are focal sensory system symptomatology as well as scatters of sensorium. Not inconsistently, such patients have other related ailments, which may alter the clinical picture exhibited by the anomalies of salt and water balance [9]. Intense hyponatremia is characterized by beginning of side effects $<48 \mathrm{hrs}$. Patients with intense hyponatremia create neurologic manifestations coming about because of cerebral edema incited by water development into the mind. These may incorporate seizures, debilitated mental status or trance like state and death. While chronic hyponatremia creating over $>48 \mathrm{hrs}$ should be considered "chronic." Most patients have chronic hyponatremia. The serum sodium focus is more often than not above $120 \mathrm{meq} / \mathrm{L} \mathrm{[10]}$.

\section{Materials and Methodology}

Place of Study: The study was done in Deccan College of Medical Sciences \& Owaisi Group of Hospitals, the facility is 1000 bedded teaching hospital, in the heart of Hyderabad in Telangana State which provides tertiary level clinical care

Study Population: Study population consisted from Infants to children aged till 12 years attending the pediatric Department both OPD and IPD care for acute gastroenteritis are enroll in the study who is satisfying the criteria for Moderate to severe dehydration.

A total of 80 Children including neonates are enrolled in the study.

Period of Study: the study was conducted from January 2018 to December2018.

Type of Study: Prospective, Observational Study

Sample collection and analysis: After obtaining informed consent from the parents of cases, they are subjected to detailed history and clinical examination and the findings are entered in the Performa. Blood samples are collected and measures of serum electrolytes \& Sr Calcium are measured and compared with that of the clinical aspects of the patient i.e diarrhea, vomiting, thirst, fever, abdominal distention and convulsions followed by urine analysis.

\section{Inclusion criteria}

1. Watery diarrhoea of 4 or more episodes per day.

2. Age group less than 12 years

3. Signs and symptoms suggestive of mild dehydration.

4. Without any other severe complications.

\section{Exclusion criteria}

1. Diarrhoea of 12 or more episodes per day.

2. Haematological disorders, chronic illness.

3. Signs suggestive of mild or no dehydration

Statistical analysis: Statistical analysis was done with EpiInfo, SPSS and Microsoft Excel.

Ethical Approval: approval from institutional review board was obtained before the study was initiated. 


\section{Original Research Article}

\section{Aims and Objectives}

1. To estimate the incidence of hyponatraemic dehydration in neonates and children aged till 12 years.
2. To evaluates the clinical features associated with acute gastroenteritis associated dehydration.

3. To determine the clinical correlates of serum electrolytes in acute gastroenteritis.

\section{Results}

80 cases were studied and analyzed to detect Gastroenteritis associated dehydration and its clinical features as a possible risk factor for hyponatremia. It was found that $60 \%$ of the males were having acute gastroenteritis while it was $40 \%$ in female subjects. The data also reveals that higher incidence of acute gastroenteritis was noted between 6 to 24 months of age while the lower incidence was found in the subjects above 36 months of age. (Table 1)

Table-1: Age and Gender Distribution of the Subjects in Study

\begin{tabular}{|c|c|c|c|c|c|c|}
\hline Age distribution & \multicolumn{2}{|c|}{ Male } & \multicolumn{2}{c|}{ Female } & \multicolumn{3}{c|}{ Total } \\
\hline & $(\mathrm{n})$ & $\%$ & $(\mathrm{n})$ & $\%$ & $(\mathrm{n})$ & $\%$ \\
\hline $6 \mathrm{~m}-24 \mathrm{~m}$ & 41 & 51.2 & 29 & 36.3 & 70 & 87.5 \\
\hline $25 \mathrm{~m}-36 \mathrm{~m}$ & 4 & 5 & 1 & 1.2 & 5 & 6.2 \\
\hline Above $36 \mathrm{~m}$ & 3 & 3.7 & 2 & 2.5 & 5 & 6.2 \\
\hline Total & $\mathbf{4 8}$ & $\mathbf{6 0}$ & $\mathbf{3 2}$ & $\mathbf{4 0}$ & $\mathbf{8 0}$ & $\mathbf{1 0 0}$ \\
\hline
\end{tabular}

Hyponatremia was occurred in 28 subjects with high incidence in lower age group in both the genders. Males (41.5\%) were more effected than females (31.2\%). (Table 2). Lowest sodium level was $127 \mathrm{mmol}$.

Table-2: Incidence of Hyponatermia with respect to age and Gender

\begin{tabular}{|c|c|c|c|c|c|c|c|c|}
\hline Age & \multicolumn{4}{|c|}{ Male } & \multicolumn{3}{c|}{ Females } \\
\hline & Hyponatermic & Normal & Total & $\begin{array}{c}\text { \% } \\
\text { affected }\end{array}$ & Hyponatermic & Normal & Total & $\begin{array}{c}\% \\
\text { affected }\end{array}$ \\
\hline $6 \mathrm{~m}-24 \mathrm{~m}$ & 15 & 26 & 41 & 31.2 & 9 & 20 & 29 & 28.1 \\
\hline $25 \mathrm{~m}-36 \mathrm{~m}$ & 2 & 2 & 4 & 8.3 & 1 & 0 & 1 & 3.1 \\
\hline $\begin{array}{c}\text { Above } \\
36 \mathrm{~m}\end{array}$ & 1 & 2 & 3 & 2 & 0 & 2 & 2 & 0 \\
\hline Total & $\mathbf{1 8}$ & $\mathbf{3 0}$ & $\mathbf{4 8}$ & $\mathbf{4 1 . 5} \%$ & $\mathbf{1 0}$ & $\mathbf{2 2}$ & $\mathbf{3 2}$ & $\mathbf{3 1 . 2 \%}$ \\
\hline
\end{tabular}

Among 80 individuals, 25 were severely dehydrated and rest of them were moderately dehydrated (55n). The number of episodes of loose stools were correlating with the extent of dehydration. Higher the episodes the severe was the dehydration. (Table 3)

Table-3: Incidence of severity of dehydration with Respect to age and gender:

\begin{tabular}{|c|c|c|c|c|c|c|c|c|}
\hline Age & \multicolumn{4}{|c|}{ Male } & \multicolumn{4}{c|}{ Females } \\
\hline & Severe & Moderate & Total & \% & Severe & Moderate & Total & $\%$ \\
\hline $6 \mathrm{~m}-24 \mathrm{~m}$ & 13 & 28 & 41 & 85.4 & 11 & 18 & 29 & 90.6 \\
\hline $25 \mathrm{~m}-36 \mathrm{~m}$ & 0 & 4 & 4 & 8.3 & 1 & 0 & 1 & 3.125 \\
\hline $\begin{array}{c}\text { Above } \\
36 \mathrm{~m}\end{array}$ & 0 & 3 & 3 & 6.2 & 0 & 2 & 2 & 6.25 \\
\hline Total & $\mathbf{1 3}$ & $\mathbf{3 5}$ & $\mathbf{4 8}$ & $\mathbf{1 0 0}$ & $\mathbf{1 2}$ & $\mathbf{2 0}$ & $\mathbf{3 2}$ & $\mathbf{1 0 0}$ \\
\hline
\end{tabular}

Vomiting were associated with diarrhea in 52 subjects whereas 28 subjects were only complained of diarrhea. (Fig 1) 
The subjects who were severely dehydrated showed both the manifestations of diarrhea and vomiting except 11 individuals.

Only 6 individuals showed hypokalemia without hyponatremia. About 3 subjects showed hypokalemia with hyponatremia (Fig 1)

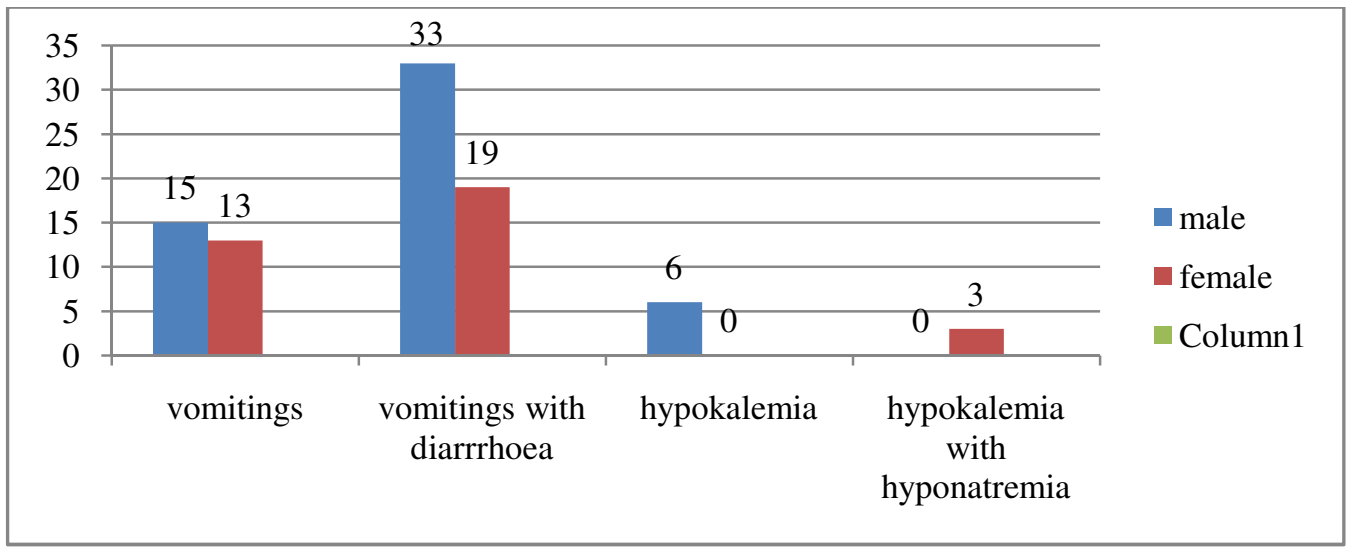

Figure-6: Distribution of clinical \& objective features

\section{Discussion}

Diarrhoea remains the second most common cause of hyponatremia in children [1]. In our study the most effected age population was 6 to 24 months and more effected were males In a study by SV Prasad et al found that $29.8 \%$ of the sick children is suffering from hyponatremia who require emergency care and must hospitalize while compared to the reported data in adult population, it also states that frequency oh hyponatremia is much higher in hospitalized sick children [1].

While our study reveals that overall $30 \%$ of the population enrol in the study suffers from hyponatremia which includes both genders from the patients admitted for the gastroenteritis. Neurological complication and symptoms arising from severe hyponatremia can be treated with $3 \%$ hypertonic saline [2] while in our study the paediatric population is treated with isotonic saline $(0.9 \%)$ with a dose of $20 \mathrm{ml} / \mathrm{kg}$ till the symptoms of the dehydration is resolved and gastroenteritis is corrected followed by oral rehydration salts, as there were no neurological manifestations in the subjects.

Diarrhoea is the most concerning cause of dehydration in children as most of the fluids during gastroenteritis are lost. Our study reveals that prolong patient stay in the hospital is due to increase in the frequency of diarrhoea and so the risk of hyponatremia. Furthermore, emesis is also an important factor contributing to the fluid loss from the body resulting in electrolyte imbalance. As shown in our study, emesis and diarrhoea leads to hypokalemia as well in few subjects [6]. To tackle this, we need to monitor potassium levels in conjunction with sodium levels. To treat dehydration in children, calculated amount of fluids need to be infused as warranted by a study which shows that the maintenance fluid need for ongoing losses and deficit needs have to be fulfilled by providing adequate fluids till normo-volemia is achieved[3].

As stated by Jacob in his study that incidence of diarrhoea is high in children particularly in developing countries, but very low proportion of the effected children visits to the clinic. Our study also supports this results that children effected with diarrhoea is less frequently hospitalized than gastroenteritis [4].

As mention in the book of Australian prescribers that approximately $5 \%$ of the outpatients and $15 \%$ of the inpatients are drug induced hyponatremia in adults [5], but in children the incidence of drug induced hyponatremia is very rare.

In our study the included population was only affected with gastroenteritis, hence there should be a broad category of diseases to be included in further research to know exactly the rare causes of hyponatremia in children.

Jagdishet.al in Turkish province found that 5.6\% neonates experience serum sodium concentrations of more than $145 \mathrm{mmol} / \mathrm{L}$ in hypernatremia dehydration [6], while our study shows that $29.8 \%$ of the infants have serum sodium levels lower than $135 \mathrm{mmol} / \mathrm{L}$ in hyponatremic dehydration. 


\section{Original Research Article}

Clinical management of hyponatremia depends on treating the fundamental causes however precise assurance of etiology of hyponatremia is not known, additionally, a clinical history might be hard to get because of pediatric population.

Hence proper history from the parents and pediatrician clinical judgment is the most broadly acknowledged methods for acquiring precise conclusion of hyponatremia and its treatment in pediatric population.

\section{Strengths}

1. Prospective Study.

2. Helps in correcting hyponatremia in gastroenteritis in paediatric population.

\section{Conclusions}

The clinical impression of the type of dehydrationand electrolyte disturbances was fairly consistent with serum electrolytes values. This suggest that routine estimation of serum electrolytes is not necessary however it is necessary whenever electrolyte imbalance is suspected on clinical grounds and in cases which do not respond satisfactorily with routine fluid electrolyte therapy.

1. The prevalence of hyponatermia indicates that the values are on the borderline, hence considering the values during the treatment of gastroenteritis will helpful in correcting theelectrolytes.

2. The Incidence of diarrhoeapeaks in children less than 1 year of age and then decreases as age increases. The analysis of clinical presentation revealed that vomiting and fever were frequent accompanied with hyponatermic dehydration.

What studyadds to existing knowledge- The present study was based on the very small sample size, hence a larger study group is recommended to come to a very definitive conclusion.

Funding: Nil, Conflict of interest: None initiated, Perission from IRB: Yes

\section{References}

1. Prasad SV, Singhi S, Chugh KS. Hyponatremia in sick children seeking pediatric emergency care. Indian Pediatr. 1994 Mar;31(3):287-94

2. Dr. Peter Allan \&Dr.SaibalGanguly, ATOTW 314 Hyponatraemia (2nd April 2015) ,Tutorial 314,Page1-8.

3. Karen S. Powers, Dehydration: Isonatremic, Hyponatremic, and Hypernatremic Recognition and Management. Pediatrics in Review, 2015 July 36 (7): 274-422

4. Jacob J, Joseph TK, Srinivasan R, et al. Direct and Indirect Costs of Pediatric Gastroenteritis in Vellore, India. Indian Pediatr. 2016 Jul 8;53(7):642-4.

5. Spiros Fourlanos, Peter Greenberg. Managing druginduced hyponatraemia in adults. Australian Prescriber, 2003 Vol. 26 (5): 114-117.

6. Das, Jagadish. (2015). Hypernatremic Dehydration in Newborn Infants: A Review. Ulutas Med J2015;1(2): 22-25.DOI:10.5455/umj.201506181158472.

7. Soiza, R. L., Cumming, K., Clarke, J. M., Wood, K. M., \& Myint, P. K. (2014). Hyponatremia: Special Considerations in Older Patients. Journal of clinical medicine, 3(3), 944-958. doi:10.3390/jcm3030944.

8. Braun MM, Barstow CH, Pyzocha NJ. Diagnosis and management of sodium disorders: hyponatremia and hypernatremia. Am Fam Physician. 2015 Mar 1;91(5): 299-307.

9. Arieff AI, Guisado R. Effects on the central nervous system of hypernatremic and hyponatremic states. Kidney Int. 1976 Jul;10(1):104-16.

10. Sahay M, Sahay R. Hyponatremia: A practical approach. Indian J Endocrinol Metab. 2014 Nov;18(6): 760-71. doi: 10.4103/2230-8210.141320.

\section{How to cite this article?}

Mohammed R, Khan M.A. A Prospective Study on the Clinical Features of Hyponatremic Dehydration in Acute Gastroenteritis.Int J Pediatr Res. 2019;6(04):166-170.doi:10.17511/ijpr.2019.i04.03 